\title{
ON THE USE OF THE INTERPOLATION POLYNOMIAL FOR SOLUTIONS OF SINGULAR INTEGRAL EQUATIONS*
}

\author{
By STEEN KRENK (Technical University of Denmark, Lyngby)
}

\begin{abstract}
On the basis of integration of singular integral equations by means of Gaussian quadrature, it is demonstrated how to obtain the corresponding approximate polynomial solution. For some special cases compact formulas are given for the strength of the singularities at the endpoints of the integration interval.
\end{abstract}

1. Introduction. For singular integral equations of the form

$$
a f(x)+\frac{b}{\pi} \int_{-1}^{1} f(t) \frac{d t}{t-x}+\int_{-1}^{1} k(t, x) f(t) d t=g(x), \quad-1<x<1,
$$

where $g(x)$ and $k(t, x)$ are known Hölder-continuous functions and $a$ and $b$ are real constants, two effective ways of numerical solution have been devised [1-4]. They both result in an approximate solution of the form

$$
f(x)=w(x) F(x) \simeq w(x) \sum_{i=0}^{n-1} c_{i} p_{i}(x),
$$

where $w(x)$ is the fundamental function of (1.1), and $p_{i}, j=0, \cdots, n-1$, are the orthogonal polynomials associated with $w(x)$. It is easily shown [5] that $w(x)$ is of the form

$$
w(x)=(1-x)^{\alpha}(1+x)^{\beta}, \quad-1<\alpha, \beta<1,
$$

and accordingly $p_{j}(x)$ are the Jacobi polynomials denoted by $P_{i}^{(\alpha, \beta)}(x)[6]$.

Although the two methods yield results of the same form, the underlying principles are different. The first method is based upon the development of $F(x)$ as an infinite series

$$
F(x)=\sum_{i=0}^{\infty} C_{i} P_{i}^{(\alpha, \beta)}(x) .
$$

By use of the orthogonality relation [6]

$$
\begin{aligned}
& \int_{-1}^{1} P_{i}^{(\alpha, \beta)}(t) P_{k}^{(\alpha, \beta)}(t)(1-t)^{\alpha}(1+t)^{\beta} d t=h_{i} \delta_{j k}, \\
& h_{i}=\frac{2^{\alpha+\beta+1}}{2 j+\alpha+\beta+1} \frac{\Gamma(j+\alpha+1) \Gamma(j+\beta+1)}{j ! \Gamma(j+\alpha+\beta+1)},
\end{aligned}
$$

an infinite system of linear equations is obtained for the determination of the coefficients $C_{i}[1,4]$. As a rule numerical evaluation of integrals must be used extensively. By trunca-

* Received October 1, 1973. This work was carried out during a visit to Lehigh University and partly supported by the National Science Foundation under Grant GK-11977. 
tion an approximate system of equations is obtained for the determination of the $n$ coefficients $C_{i}, j=0,1, \cdots, n-1$.

The second method starts with an approximating polynomial of finite degree,

$$
F(x) \simeq \sum_{i=0}^{n-1} c_{i} P_{i}^{(\alpha, \beta)}(x) .
$$

By applying the quadrature formulas given in [2] and [3], approximate values of $F(x)$ are obtained in the points $x_{i}$ determined by $P_{n}{ }^{(\alpha, \beta)}\left(x_{i}\right)=0, i=1,2, \cdots, n^{*}$. It is important to note that this is accomplished without performing numerical evaluation of integrals.

The present paper gives simple summation formulas for the determination of the coefficients $c_{i}$ in (1.7). For the important cases $(\alpha, \beta)=( \pm 1 / 2, \pm 1 / 2)$, which correspond to $a=0$, special formulas are derived in terms of Chebyshev polynomials. These include formulas for direct determination of $F(1)$ and $F(-1)$.

2. General formulas. Polynomials orthogonal over a finite interval satisfy a recurrence relation which may be put in the form $[6,8]$

$$
a_{i-1} p_{i-1}(x)+b_{i} p_{j}(x)+a_{i} p_{i+1}(x)=x p_{j}(x) .
$$

In the following, $p_{i}(x), j=0,1, \cdots, n-1$, will be considered as normalized. By choosing $x$ as

$$
p_{n}\left(x_{i}\right)=0, \quad i=1,2, \cdots, n,
$$

and defining $p_{-1}(x) \equiv 0$, (2.1) may be written using a symmetrical tridiagonal matrix

$$
\left[\begin{array}{cccccc}
b_{0} & a_{0} & & & & \\
a_{0} & b_{1} & a_{1} & & & \\
& a_{1} & b_{2} & a_{2} & & \\
& & & a_{n-3} & b_{n-2} & a_{n-2} \\
& & & & a_{n-2} & b_{n-1}
\end{array}\right]\left[\begin{array}{l}
p_{0}\left(x_{i}\right) \\
p_{1}\left(x_{i}\right) \\
p_{2}\left(x_{i}\right) \\
\\
p_{n-1}\left(x_{i}\right)
\end{array}\right]=x_{i}\left[\begin{array}{c}
p_{0}\left(x_{i}\right) \\
p_{1}\left(x_{i}\right) \\
p_{2}\left(x_{i}\right) \\
\\
p_{n-1}\left(x_{i}\right)
\end{array}\right] .
$$

The vectors $\left[p_{0}\left(x_{i}\right), p_{1}\left(x_{i}\right), \cdots, p_{n-1}\left(x_{i}\right)\right], i=1,2, \cdots, n$, are eigenvectors corresponding to the eigenvalues $x_{i}$. These vectors are normalized by calculating

$$
\sum_{i=0}^{n-1} p_{i}^{2}\left(x_{i}\right)=\frac{1}{\lambda_{i n}} \text {. }
$$

By using the Christoffel-Darboux formula [6], the $\lambda_{i n}, i=1,2, \cdots, n$, are seen to be the weights from the corresponding Gaussian quadrature formula. As the matrix $\left\{\sqrt{\lambda_{i n}} p_{j}\left(x_{i}\right)\right\}$ is orthogonal with respect to both rows and columns, we obtain the formula

$$
\sum_{i=1}^{n} \lambda_{i n} p_{i}\left(x_{i}\right) p_{k}\left(x_{i}\right)=\delta_{i k}
$$

Just as $C_{i}, j=0,1, \cdots$ are determined by the continuous orthogonality relation, $c_{i}, j=0,1, \cdots, n-1$, are determined by use of the discrete orthogonality relation (2.5). The result is

\footnotetext{
${ }^{*}$ For $\alpha=\beta= \pm 1 / 2$ the connection between $C_{j}$ and $c_{j}$ is discussed in [7].
} 


$$
F(x) \simeq \sum_{i=0}^{n-1} c_{i} p_{i}(x), \quad c_{i}=\sum_{i=1}^{n} \lambda_{i n} p_{i}\left(x_{i}\right) F\left(x_{i}\right)
$$

For the Jacobi polynomials (2.6) is slightly modified:

$$
F(x) \simeq \sum_{i=0}^{n-1} c_{i} P_{i}^{(\alpha, \beta)}(x), \quad c_{i}=\frac{1}{h_{i}} \sum_{i=0}^{n} \lambda_{i n} P_{i}^{(\alpha, \beta)}\left(x_{i}\right) F\left(x_{i}\right),
$$

where $h_{i}$ is given by $(1.6)$ and $\lambda_{i n}$ by $[3,4]$

$$
\lambda_{i n}=-2^{\alpha+\beta} \frac{\Gamma(n+\alpha+1) \Gamma(n+\beta+1)}{(n+1) ! \Gamma(n+\alpha+\beta+2)} \frac{2 n+\alpha+\beta+2}{P_{n}^{(\alpha, \beta) \prime}\left(x_{i}\right) P_{n+1}{ }^{(\alpha, \beta)}\left(x_{i}\right)} .
$$

3. Special cases. The cases $(\alpha, \beta)=( \pm 1 / 2, \pm 1 / 2)$ are special because the corresponding Jacobi polynomials can be expressed by Chebyshev polynomials. This gives the possibility of direct determination of $x_{i}$ from (2.2) by means of trigonometric functions [4]. Furthermore, the index $j$ may be summed analytically in the calculation of $F(1)$ and $F(-1)$. These values are of special physical importance, for example, in evaluation of stress intensity factors [4] and [9-11].

In the following the summation sign $\sum^{\prime}$ means that the first term must be multiplied by $1 / 2$. Use of $\sum^{\prime \prime}$ implies that both first and last terms must be multiplied by $1 / 2$. The derivation of the formulas is based on the trigonometric definition of the Chebyshev polynomials and the two relations

$$
\begin{aligned}
& \sum_{i=0}^{n} \prime \prime \cos (j \theta)=\frac{1}{2} \sin (n \theta) \cot \left(\frac{1}{2} \theta\right), \\
& \sum_{i=0}^{n-1} \cos [(2 j+1) \theta]=\frac{1}{2} \frac{\sin (2 n \theta)}{\sin \theta} . \\
& \alpha=\beta=-1 / 2 \text { : } \\
& F(x) \simeq \sum_{j=0}^{n-1} c_{i} T_{i}(x), \\
& c_{i}=\frac{2}{n} \sum_{i=1}^{n} T_{j}\left(x_{i}\right) F\left(x_{i}\right)=\frac{2}{n} \sum_{i=1}^{n} \cos \left[\frac{2 i-1}{2 n} j \pi\right] F\left(x_{i}\right), \\
& x_{i}=\cos \left[\frac{2 i-1}{2 n} \pi\right] \text {. } \\
& F(1) \simeq \frac{1}{n} \sum_{i=1}^{n} U_{2 n-2}\left[\left(1+x_{i}\right) / 2\right]^{1 / 2} F\left(x_{i}\right) \\
& \frac{1}{n} \sum_{i=1}^{n} \frac{\sin \left[\frac{2 n-1}{4 n}(2 i-1) \pi\right]}{\sin \left[\frac{2 i-1}{4 n} \pi\right]} F\left(x_{i}\right) \text {. } \\
& F(-1) \simeq \frac{1}{n} \sum_{i=1}^{n} U_{2 n-2}\left[\left(1-x_{i}\right) / 2\right]^{1 / 2} F\left(x_{i}\right) \\
& =\frac{1}{n} \sum_{i=1}^{n} \frac{\sin \left[\frac{2 n-1}{4 n}(2 i-1) \pi\right]}{\sin \left[\frac{2 i-1}{4 n} \pi\right]} F\left(x_{n+1-i}\right) .
\end{aligned}
$$


$\alpha=\beta=1 / 2$ :

$$
\begin{aligned}
F(x) & \simeq \sum_{i=0}^{n-1} c_{i} U_{i}(x) \\
c_{i} & =\frac{2}{n+1} \sum_{i=1}^{n}\left(1-x_{i}{ }^{2}\right) U_{i}\left(x_{i}\right) F\left(x_{i}\right) \\
& =\frac{2}{n+1} \sum_{i=1}^{n} \sin \left[\frac{i}{n+1} \pi\right] \sin \left[\frac{i}{n+1}(j+1) \pi\right] F\left(x_{i}\right), \\
x_{i} & =\cos \left[\frac{i}{n+1} \pi\right] . \\
F(1) & \simeq \sum_{i=1}^{n}\left(1+x_{i}\right) U_{n-1}\left(x_{i}\right) F\left(x_{i}\right) \\
& =\sum_{i=1}^{n} \cot \left[\frac{i}{n+1} \frac{\pi}{2}\right] \sin \left[\frac{n}{n+1} i \pi\right] F\left(x_{i}\right) . \\
F(-1) & \simeq \sum_{i=1}^{n}\left(1-x_{i}\right) U_{n-1}\left(-x_{i}\right) F\left(x_{i}\right) \\
& =\sum_{i=1}^{n} \cot \left[\frac{i}{n+1} \frac{\pi}{2}\right] \sin \left[\frac{n}{n+1} i \pi\right] F\left(x_{n+1-1}\right) .
\end{aligned}
$$

$\alpha=-\beta=1 / 2$ :

$$
\begin{aligned}
& F(x) \simeq \sum_{i=0}^{n-1} c_{i} U_{2 i}[(1+x) / 2]^{1 / 2}, \\
& c_{i}=\frac{2}{2 n+1} \sum_{i=1}^{n}\left(1-x_{i}\right) U_{2 i}\left[\sqrt{\left(1+x_{i}\right) / 2}\right] F\left(x_{i}\right) \\
& =\frac{4}{2 n+1} \sum_{i=1}^{n} \sin \left[\frac{i}{2 n+1} \pi\right] \sin \left[\frac{i}{2 n+1}(2 j+1) \pi\right] F\left(x_{i}\right) \text {. } \\
& x_{i}=\cos \left[\frac{2 i}{2 n+1} \pi\right] \text {. } \\
& F(1) \simeq \sum_{i=1}^{n} U_{2 n-2}\left[\sqrt{\left(1+x_{i}\right) / 2}\right] F\left(x_{i}\right) \\
& =\sum_{i=1}^{n} \frac{\sin \left[\frac{2 n-1}{2 n+1} i \pi\right]}{\sin \left[\frac{i}{2 n+1} \pi\right]} F\left(x_{i}\right) \\
& F(-1) \simeq \frac{2}{2 n+1} \sum_{i=1}^{n} \sqrt{\left(1-x_{i}\right) / 2} U_{2 n-1}\left[\sqrt{\left(1-x_{i}\right) / 2}\right] F\left(x_{i}\right) \\
& =\frac{2}{2 n+1} \sum_{i=1}^{n} \cot \left[\frac{2 i-1}{2 n+1} \frac{\pi}{2}\right] \sin \left[\frac{n}{2 n+1}(2 i-1) \pi\right] F\left(x_{n+1-i}\right) \text {. }
\end{aligned}
$$


$\alpha=-\beta=-1 / 2$ :

$$
\begin{aligned}
F(x) & \simeq \sum_{i=0}^{n-1} c_{i} \sqrt{2 /(1+x)} T_{2 i+1}[\sqrt{(1+x) / 2}] \\
c_{i} & =\frac{4}{2 n+1} \sum_{i=1}^{n} \sqrt{\left(1+x_{i}\right) / 2} T_{2 i+1}\left[\sqrt{\left(1+x_{i}\right) / 2}\right] F\left(x_{i}\right) \\
& =\frac{4}{2 n+1} \sum_{i=1}^{n} \cos \left[\frac{2 i-1}{2 n+1} \frac{\pi}{2}\right] \cos \left[\frac{2 i-1}{2 n+1}(2 j+1) \frac{\pi}{2}\right] F\left(x_{i}\right), \\
x_{i} & =\cos \left[\frac{2 i-1}{2 n+1} \pi\right] . \\
F(1) & \simeq \frac{2}{2 n+1} \sum_{i=1}^{n} \sqrt{\left(1+x_{i}\right) / 2} U_{2 n-1}\left[\sqrt{\left(1+x_{i}\right) / 2}\right] F\left(x_{i}\right) \\
& =\frac{2}{2 n+1} \sum_{i=1}^{n} \cot \left[\frac{2 i-1}{2 n+1} \frac{\pi}{2}\right] \sin \left[\frac{n}{2 n+1}(2 i-1) \pi\right] F\left(x_{i}\right) . \\
F(-1) & \simeq \sum_{i=1}^{n} U_{2 n-2}\left[\sqrt{\left(1-x_{i}\right) / 2}\right] F\left(x_{i}\right) \\
& =\sum_{i=1}^{n} \frac{\sin \left[\frac{2 n-1}{2 n+1} i \frac{i}{\sin }\left[\frac{i}{2 n+1} \pi\right]\right.}{F\left(x_{n+1-i}\right)}
\end{aligned}
$$

4. Example. The equation for this example is taken from [2]:

$$
\frac{1}{\pi} \int_{-1}^{1} \frac{f(t)}{t-x} d t-\lambda \int_{-1}^{x} f(t) d t=1, \quad-1<x<1 .
$$

$f(x)$ is the shear stress acting on the interface between an elastic coverplate and an elastic half-space, when the half-space is loaded parallel to the plate at infinity. Of special interest is the stress intensity factor at $x=1$ represented by $F(1)$. In [2] values of $F(1)$ were obtained by three-point extrapolation for $\lambda=10 / 3,1 / 3$, and $n=20,40$, 60 . In Table 1 these values are given together with the similar values obtained by use of (3.4). The difference is not great, a fact that follows from the smoothness of $F(x)$. That greater differences must be expected for less smooth functions is clearly seen from the weights in (3.4), which are given in Table 2 for $n=20$. In particular, when inferences are made from the values of $F(1)$ concerning the convergence of the method of quadrature, the formulas presented here must be used.

TABLE 1

The strength of the stress singularity, $F(1)$.

\begin{tabular}{ccccc}
\hline$F(1)$ & \multicolumn{2}{c}{$\lambda=10 / 3$} & \multicolumn{2}{c}{$\lambda=1 / 3$} \\
\hline$n$ & {$[1]$} & $(3.4)$ & {$[1]$} & $(3.4)$ \\
\hline 20 & 0.4061 & 0.4076 & 0.8323 & 0.8325 \\
40 & 0.4104 & 0.4108 & 0.8331 & 0.8332 \\
60 & 0.4115 & 0.4117 & 0.8340 & 0.8341 \\
\hline
\end{tabular}


TABLE 2

Weights for the calculation of $F(1)$ for $\alpha=\beta=-1 / 2$ and $n=20$ (3.4).

\begin{tabular}{rrrr}
\hline$i$ & $U_{2 n-2}\left(\left(1+x_{i}\right) / 2\right)^{1 / 2}$ & $i$ & $U_{2 n-2}\left(\left(1+x_{i}\right) / 2\right)^{1 / 2}$ \\
\hline 1 & 25.4517 & 11 & 0.9244 \\
2 & -8.4490 & 12 & -0.7883 \\
3 & 5.0273 & 13 & 0.6682 \\
4 & -3.5457 & 14 & -0.5600 \\
5 & 2.7106 & 15 & 0.4610 \\
6 & -2.1692 & 16 & -0.3689 \\
7 & 1.7856 & 17 & 0.2820 \\
8 & -1.4966 & 18 & -0.1989 \\
9 & 1.2685 & 19 & 0.1184 \\
10 & -1.0818 & 20 & -0.0393 \\
\hline
\end{tabular}

\section{REFERENCES}

[1] F. Erdogan, Approximate solutions of systems of singular integral equations, SIAM J. Appl. Math. 17, 1041 (1969)

[2] F. Erdogan and G. D. Gupta, On the numerical solution of singular integral equations, Quart. Appl. Math. 30, 525 (1972)

[3] S. Krenk, On quadrature formulas for singular integral equations of the first and the second kind, Lehigh University, Report IFSM-73-47, August 1973; Quant. Appl. Math., to appear

[4] F. Erdogan, G. D. Gupta and T. S. Cook, Numerical solution of singular integral equations, in Methods of analysis and solutions of crack problems, ed. G. C. Sih, Noordhoff International Publishing, Leyden, 1973

[5] N. I. Muskhelishvili, Singular integral equations, Wolters-Noordhoff Publishing, Groningen, 1958 (see $\$ 107$ )

[6] A. Erdelyi, Higher transcendental functions, Vol. 2, McGraw-Hill, New York, 1953

[7] C. Lanczos, Trigonometric interpolation of empirical and analytical functions, J. Math. Phys. 17, 123 (1938)

[8] C. Lanczos, Applied analysis, Prentice-Hall, New York, 1957

[9] F. Erdogan and G. D. Gupta, Stresses near a flat inclusion in bonded dissimilar materials, Int. J. Solids Structures 8, 533 (1972)

[10] F. Erdogan and M. Ratwani, A circumferential crack in a cylindrical shell under torsion, Int. J. Fracture Mech. 8, 87 (1972)

[11] U. Yuceoglu and F. Erdogan, A cylindrical shell with an axial crack under skew-symmetric loading, Int. J. Solids Structures 9, 347 (1973) 